\title{
Análisis de la movilidad estudiantil internacional a nivel pregrado: Un caso de estudio
}

\section{Analysis of international student mobility at the undergraduate level: A case study}

\author{
Omar Gustavo Guillén Velastegui ${ }^{1, *}$ \\ ${ }^{1}$ Facultad de Hospitalidad - Dirección de Relaciones Internacionales, Universidad de Cuenca, Ecuador. \\ gustavo.guillen@ucuenca.edu.ec
}

Fecha de recepción: 10 de noviembre de 2017 — Fecha de aceptación: 11 de diciembre de 2017

DOI: https://doi.org/10.26910/issn.2528-8083vol2iss8.2017pp37-43

\begin{abstract}
Resumen-El presente trabajo de investigación da a conocer el análisis de la movilidad estudiantil internacional a nivel de pregrado generada en la Universidad de Cuenca- Ecuador entre instituciones de educación superior durante los años 2015 y 2016 , cabe señalar que los procesos formales de intercambio estudiantil favorecen en la formación académica de estudiantes, y las Instituciones de Educación Superior (IES) juegan un rol fundamental para su ejecución; y consecuentemente deben mantenerse en comunicación, utilizando herramientas tecnológicas capaces de responder a las necesidades actuales que requieren de respuestas inmediatas, manteniendo su nivel de calidad. El proyecto planteó analizar las políticas vigentes de la instancia responsable de la movilidad estudiantil, identificando las áreas del conocimiento que interesan los estudiantes extranjeros, la dinámica de movilidad de los países de procedencia según los periodos académicos del estudio.En base a los resultados obtenidos se recomendaron algunas políticas para la dependencia, con criterios que permitieron establecer estrategias para mejorar los procesos de movilidad estudiantil que optimicen las actividades de los programas e incrementar la cooperación entre las IES en América, Europa y Asia.
\end{abstract}

Palabras Clave-Movilidad Estudiantil, Políticas, Instituciones de Educación Superior, Relaciones Internacionales.

\begin{abstract}
This research work reveals the analysis of international undergraduate student mobility generated at the University of Cuenca-Ecuador between higher education institutions during the years 2015 and 2016. It should be noted that the formal processes of student exchange in favor of the academic formation of students, and the Institutions of Higher Education (IHE) has a fundamental role in its execution; and consequently, it must keep in communication, using technological tools capable of responding to current needs that require immediate answers, maintaining a proper level of quality. The project proposed to analyze the current policies of the organism responsible for student mobility, identifying the areas of knowledge of interest for exchange student, and the mobility dynamics of the countries of origin according to the academic periods of the study.Based on the obtained results, some policies were recommended for the dependency, with criteria that allowed establishing strategies to improve student's mobility procedures that optimize programs activities and increase the cooperation among (IHE) in the Americas, Europe and Asia.
\end{abstract}

Keywords -Student Mobility, Policies, Higher Education Institutions, International Relations

\section{INTRODUCCIÓN}

$\mathbf{P}$ artiendo de la Declaración Universal de los Derechos Humanos Art. 13 señala que, toda persona tiene derecho a circular libremente y a elegir su residencia en el territorio de un Estado. Toda persona tiene derecho a salir de cualquier país, incluso del propio, y a regresar a su país (Organización de Naciones Unides, 1948), por lo tanto, insta a las personas a desplazarse de su lugar de origen a otro, y en el caso específico de una movilidad estudiantil, se convierte en una expresión natural en busca de una oportunidad para encontrar una educación superior de calidad. Adicional a ello, la Organización de las Naciones Unidas para la Educación, la Ciencia y la Cultura, con sus siglas en inglés (UNESCO) y otras organizaciones que actúan en el campo de la educación superior, en beneficio de la cooperación bilateral y multilateral, en conjunto con la comunidad universitaria y todos sus actores interesados en el desarrollo de la sociedad, se comprometen en promover la movilidad académica como medio de progreso de saberes; de esta manera surgen organismos internacionales que permiten

*Ingeniero en Turismo mantener una dinámica de la movilidad, tal como el Instituto Internacional de la UNESCO para la Educación Superior en América Latina y el Caribe (IESALC) el mismo que contribuye a garantizar la pertinencia, eficiencia y equidad de la educación superior con un enfoque regional e internacional, ya que, al ser considerado como el laboratorio de ideas, con una visión regional de la Educación Superior ha fortalecido las tendencias académicas, y es el puente para crear una cooperación adecuada comprometida con los intereses colectivos de sus miembros; el mismo que formula los planes, proyectos y programas con dinámicas de servicio, ideas y análisis sobre la Educación Superior de la región, de América Latina y el Caribe (IESALC, 2017); de la misma manera el Observatorio sobre movilidad académica y científica en América Latina y el Caribe (OBSMAC) autorizado por la UNESCO, resulta ser un proyecto de alcance regional, acordado en la Conferencia Regional sobre Educación Superior en América Latina y el Caribe (2008 en Cartagena de Indias, Colombia) y autorizado por la Conferencia Mundial sobre Educación Superior de la UNESCO (2009, París, Francia). El proyecto, fue puesto en funcionamiento en el año 2010, bajo la coordinación del 
IESALC, como respuesta a las preocupaciones manifestadas por las autoridades de la Educación Superior; con lo cual los análisis de movilidad académica científica obtenido del observatorio, que permiten tener una visión integral de la dinámica de la movilidad generada; al ser una amplia área heterogénea, que obtiene una gran cantidad de información. Entre algunos de los puntos que se pueden obtener de estos análisis pueden ser comprendidos como: fuga de cerebros, integración académica institucional, referentes políticos, tendencias en la educación superior, entre otros.

Entre uno de los principales referentes de procesos de internacionalización y de estrategias de cooperación educativa, la movilidad académica es uno de los pilares fundamentales de la colaboración entre instituciones y estructuras gubernamentales; también se constituye como elemento importante del conjunto de políticas educativas en materia de internacionalización de la educación superior y colaboración en materia de desarrollo. (Palma, 2013). Debido a ello, cada individuo que participa de una movilidad académica, ya sea este, estudiante, docente, investigador o administrativo de una IES, ejecuta uno de los varios objetivos de una cooperación adecuada, debido a que el proceso de internacionalización a más de la firma de un memorándum de entendimiento o un convenio de cooperación, son el soporte formal en el histórico de colaboración que guarda estos lazos de intenciones académicas y de investigación, que fomentan la integración de saberes para establecer una fluida participación de las partes involucradas.

Es conveniente indicar que, las redes y asociaciones internacionales, funcionan como puentes para trabajar en proyectos específicos o programas de interés común, de ahí la importancia del rol de autoridades institucionales en el desarrollo operativo y de gestión de programas de cooperación e intercambio institucional, ahora bien, a las puertas de la Conferencia Regional de Educación Superior (CRES 2018) en Córdoba, Argentina, evento que permitirá tener una perspectiva más amplia de una movilidad académica dada de la mano, con el uso de herramientas tecnológicas; que está ligada a una negociación de recursos y obtención de becas destinadas para movilidad, ya que la expectativa es alta ante una demanda cada vez más competitiva y mejor preparada. Adicional a ello, Juan Pablo Viscardo señala que: El Nuevo Mundo es nuestra patria, y su historia es la nuestra, y en ella es que debemos examinar nuestra situación presente, para determinarnos, por ella, a tomar el partido necesario a la conservación de nuestros derechos propios y de nuestros sucesores (Vizcardo et al., 2004). Con esta premisa, se afianza el interés del Viejo continente por establecer cooperación con América, sin embargo, en aquella época, todo era una ideología que ahora se ve mucho más delimitada, en donde claramente se puede observar que la movilidad no es el fin de las relaciones internacionales sino un producto más dentro de la gestión realizada.

Al complementarse las IES que buscan el mismo fin de mejorar la calidad de sus academias y consolidar trabajos en áreas de interés, la conformación de redes y asociaciones, permiten tener acceso a los beneficios de una cooperación más organizadas con mayores oportunidades en donde las políticas, normativas y reglamentos aplicados por estudiantes, docentes e investigadores. La relevancia de este particular, marca la importancia que tienen las Universidades para el desarrollo económico y social de las naciones, ya que sus estudiantes, son quienes innovaran y mejoraran la calidad de vida de las personas; $y$ en el proceso de una movilidad son un dinamizador económico y cada uno se convierte en embajador de su país, obteniendo así un intercambio cultural y de saberes, integrando regiones de manera natural.

Las Universidades, en miras de obtener un proceso de internacionalización, ven a la movilidad académica como una oportunidad que beneficia entre cooperantes, y que cada IES o institución con la que se plantea este trabajo debe manejar procesos reglamentados que cumplan con ciertos parámetros, para su escogimiento, la información obtenida de las correspondientes unidades académicas, deben guardarse con criterios de confidencialidad; ya sean instituciones públicas o privadas, los procesos son transparentes y claros, con una óptica profesional y crítica. Los reglamentos de movilidad académica, institucional, deberán permitir un traslado de personas regular, y sobre todo que cubra las necesidades de los postulantes, respaldados por su institución emisora. En realidad, todos los actores que participan en el proceso de movilidad, estudiantes, docentes y administrativos; al finalizar sus actividades, regresan a su institución emisora, la misma que evaluará su desempeño y permitirá en base los objetivos proponer estrategias y táctica puntuales para un mejoramiento de las IES, como así también evaluar las carreras y facultades, intereses que favorezcan a una toma de decisiones e incluso, llegar a generar un movimiento masivo y constante que se pueda consolidar, brindando beneficio s a múltiples personas, en este contexto, la cooperación internacional bien enfocada, según las necesidades institucionales son parte fundamental para generar buenas políticas centradas en el bien común entre instituciones.

Según Muñoz Varela (2017), "la movilidad estudiantil, está claramente situada en la política y en el que hacer institucional de la universidad" (Muñoz Varela, 2017). Es así que, la movilidad estudiantil de la Universidad de Cuenca debe estar estructurada de una manera tal que, sea visible y constante. Con lo cual se identificó que la estructura administrativa es un pilar fundamental protagónico de función y de gestión ya que adicionalmente debe cubrir un rol académico y de soporte para los estudiantes. Esto quiere decir que las estrategias institucionales deben estar enfocadas a la producción de una gestión programada, capaces de fortalecer vías de comunicación y de intercambio de información.

\section{Metodología}

La situación actual en el Ecuador, dado los regímenes académicos e instancias superiores que controlan y acreditan la educación superior en el país, señalan ciertos factores de los condicionantes de la movilidad, sin embargo, a nivel de pregrado es un tema aun ideológico, tal como se señaló en el Primer Encuentro de Movilidad Académica y Científica en Ecuador, organizado por la Universidad Técnica Particular de Loja (UTPL) y el Observatorio Nacional Temático del Ecuador (OBNAT-EC) en el año 2013 (Secretaria de Educación 
Superior, Ciencia, 2013); recordando que es la línea base de la cooperación internacional; en el caso de países europeos lo instauran dentro de los requerimientos para la obtención de un título de grado.

La Universidad de Cuenca se han beneficiado de una movilidad estudiantil en diferentes programas, y el número de aspirantes es cada vez mayor, además amplía y brinda cierta ventaja competitiva, y esto se ve reflejado en el mejoramiento in-situ de cada una de las facultades dentro de su perfil académico; sin embargo, diversos factores afectan a este proceso, por ejemplo; comunicación interna entre unidades académicas, políticas de movilidad, reglamentos vigentes de matrícula, etc. Este análisis favoreció para conocer el estado en el que se encontró la movilidad estudiantil internacional de estudiantes extranjeros hacia la Universidad de Cuenca; consideraron pertinentes los años 2015 y 2016; de esta forma se desarrolló una propuesta de estrategias sobre políticas institucionales en el marco de la internacionalización.

La Dirección de Relaciones Internacionales (DRI) de la Universidad de Cuenca, se basa en el Reglamento de Movilidad Estudiantil, Reconocimiento u Homologación de Estudios de la Universidad de Cuenca, en donde permite una movilidad de estudiantes que provengan de otra institución de educación superior cumpliendo ciertos requisitos, entre ellos haber cumplido primer y segundo nivel de su carrera de pregrado (Universidad de Cuenca, 2016), pero más aún, en el Art. 5 del mismo reglamento, señala que un estudiante que provenga de una Universidad sea nacional o internacional, deberá rendir un examen para el cambio de carrera, si su interés es el de empezar sus estudios de tercer nivel en la Universidad de Cuenca.

Los datos para este estudio fueron obtenidos de la DRI y para determinar la pertinencia se consideró la población de estudiantes de la Universidad de Cuenca en el año 2015 fue de 17.110 estudiantes y en el 2016 fue de 15.294 respectivamente. (Universidad de Cuenca, 2016). El número total de estudiantes de movilidad en los dos años del análisis fue de 587, sin embargo 378 estudiantes aplicaron al programa de español para extranjeros de los cuales no se ha obtuvo información. El universo considerado para este estudio es de 209 estudiantes de movilidad que aplicaron entre los años 2015 y 2016.

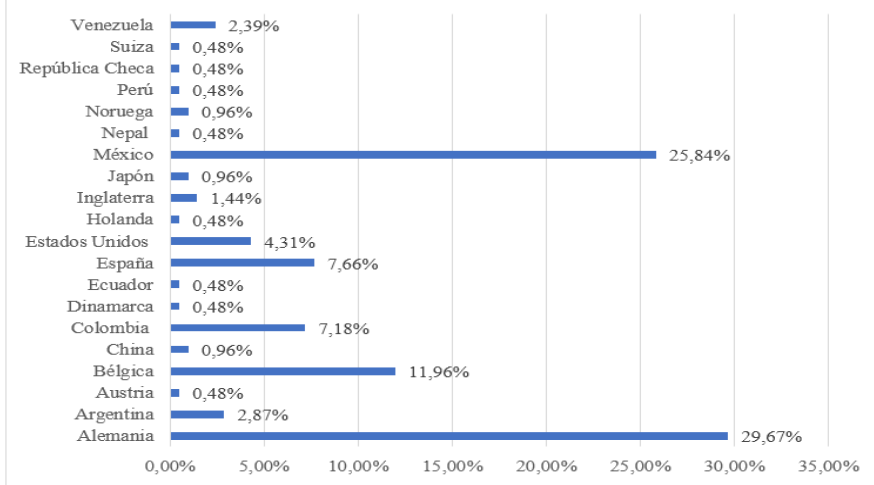

Figura 1. Procedencia

Fuente: Elaboración Propia.
La mayoría de los estudiantes de movilidad entrante corresponden a nacionalidad alemana con un $29,67 \%$; mexicanos $25,84 \%$; belgas $11,96 \%$; españoles $7,66 \%$ y colombianos $7,18 \%$; el 36,2\% corresponden a los demás países; siendo asî el mayor porcentaje de estudiantes extranjeros son europeos con un $54,07 \%$, americanos $43,54 \%$ y asiáticos el $2,39 \%$, como se puede apreciar en la Figura 1 y 2.

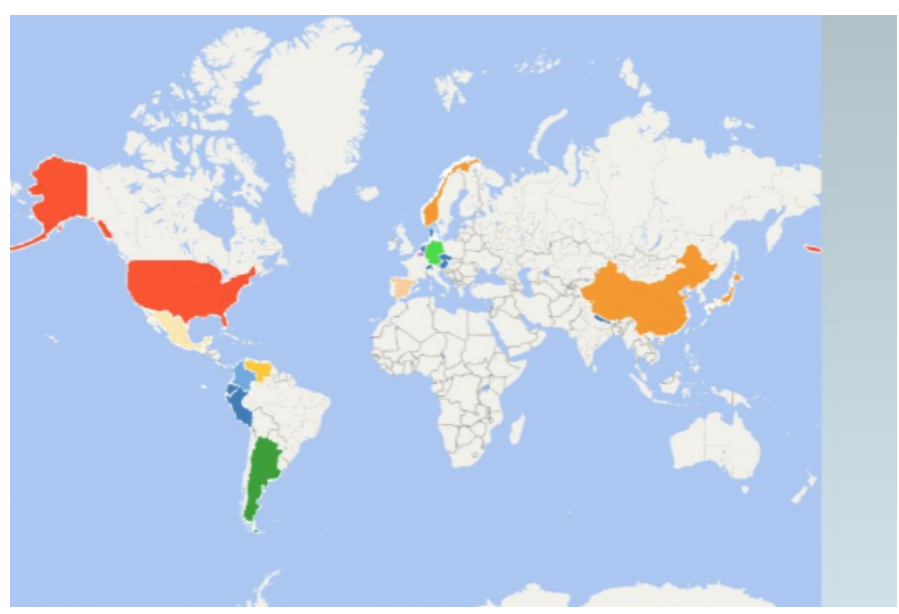

Figura 2. País de procedencia

Fuente: Elaboración Propia.

Los datos muestran que el área de la salud y servicios sociales predomina frente las demás áreas del conocimiento, en donde se registró que 79 estudiantes de pregrado estuvieron realizado sus actividades de manera regular en la institución; mientras que 45 estudiantes extranjeros estuvieron cursando sus estudios en el área de las Ciencias sociales, educación comercial y derecho, y mínimamente se tiene a 2 estudiantes que interesan por el área de agropecuaria, como se puede apreciar en la Figura 3. Se pueden observar las fortalezas y oportunidades que se tienen institucionalmente para reforzar de alguna manera el interés de estudiantes que apliquen en las áreas de menor incidencia actualmente.

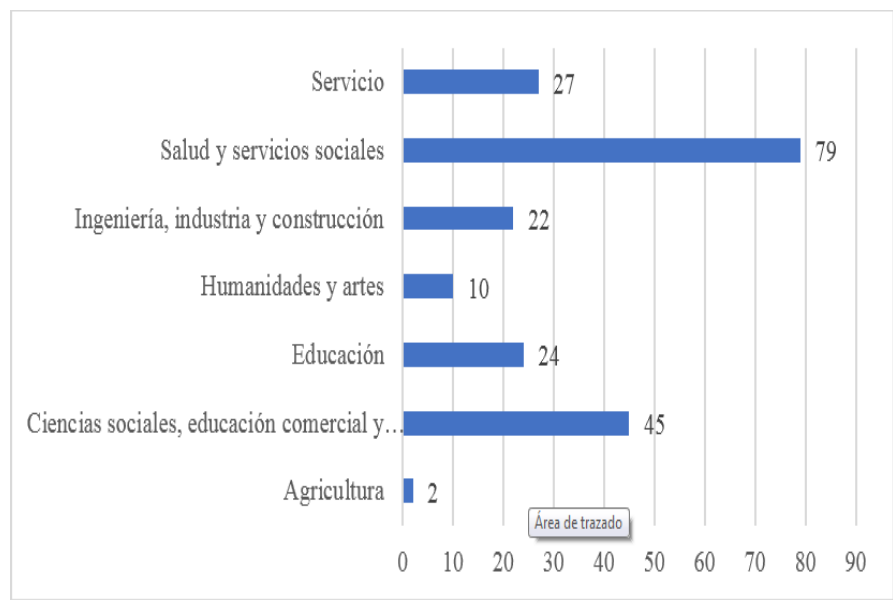

Figura 3. Movilidad estudiantil según áreas del conocimiento dadas por la UNESCO

Fuente: Elaboración Propia. 
Las rotaciones médicas son en alto porcentaje las actividades académicas más demandantes con un $34,45 \%$, que aplicaron a prácticas en el Hospital Vicente Corral Moscoso, o en sus rotaciones dentro de la zonal 6; las Facultades de Ciencias de la Hospitalidad y a la Facultad de Filosofía, Letras y Ciencias de la Educación, ambas poseen un porcentaje igual al 11,48 por bando; lo cual hace pensar que una fortalezas académica incurre dentro del áreas de la salud como en ciencias sociales, educación, comercio y derecho; seguidos de la Facultad de Jurisprudencia, Ciencias Políticas y Sociales a más de la Facultad de Psicología que son igual de apetecidos por los estudiantes extranjeros, sus porcentajes son relativamente iguales con un $7,66 \%$ y $7,18 \%$.

Comparando el año 2015, se refleja el porcentaje más alto de movilidad estudiantil en rotaciones médicas con un 44,64\%, fueron 50 estudiantes extranjeros que tuvieron la oportunidad de realizar sus pasantías en la ciudad de Cuenca. Por otro lado, la Facultad de Ciencias Económicas y Administrativas recibió 9,82\% seguido por la Facultad de Ciencias del a Hospitalidad con un $8,93 \%$. Y en el 2016 se percibió una caída en el flujo de estudiantes, las rotaciones médicas con un $23 \%$ recibiendo 22 estudiantes. La Facultad de Filosofía, Letras y Ciencias de la Educación, $16 \%$, seguida de la Facultad de Ciencias de la Hospitalidad con $14 \%$. La Universidad de Cuenca, con el afán de afianzar su cooperación, mantiene convenios de cooperación marco y específico, en donde recibió un $69 \%$ de estudiantes mediante convenios de cooperación y la diferencia del $31 \%$ sin convenio. Es importante señalar que los periodos o semestres académicos en la Universidad de Cuenca son, Septiembre - Febrero y Marzo - Julio; el mes de agosto es el mes de vacaciones para estudiantes.

Para el levantamiento de datos se aplicaron encuestas en idioma español, y se recolecto información de relevancia realizadas vía correo electrónica, estructurada en 6 bloques de preguntas: Información general, datos generales, información académica, pre movilidad, movilidad, post movilidad y recomendaciones y sugerencias. El tamaño de la muestra se aplicó de la fórmula (1) tomada del libro de Probabilidad y estadística para ingeniería.

$$
\begin{gathered}
n=\frac{N \cdot Z_{a}^{2} \cdot p(1-p)}{e^{2}(N-1)+Z_{a}^{2} \cdot p(1-p)}(1) \\
n=136
\end{gathered}
$$

$\mathrm{N}=$ Tamaño de la población $=209$

$Z^{2}=$ nivel de confianza (constante) $=95 \%$

$\mathrm{n}=$ Tamaño de la muestra

$\mathrm{e}=$ error muestral $=5 \%$

Fuente: Elaboración Propia.

De una población de 209 estudiantes de movilidad, con nivel de confianza en la fórmula es de $95 \%$ que es igual al valor estándar, la variable $\mathrm{p}$ que es la proporción de estudiantes de movilidad de la población con el $50 \%$, mientras que el error estadístico que es del $5 \%$ resultando una muestra total de 136 encuestas para cumplir el objetivo de este estudio. Adicional a ello es importante enfatizar que para la tabulación de los datos y el cruce de las variables se utilizó el programa SPSS 15.0 para Windows, herramienta estadística que facilita la elaboración de gráficos de descripción de frecuencia, Microsoft Excel 2012 y Google Forms. Del total de respuestas se obtuvo un $77 \%$ son de sexo femenino, $21 \%$ sexo masculino y un $2 \%$ prefiere no indicar su tendencia sexual; en relación a las edades de los estudiantes que aplican su movilidad, el promedio encontrado es de 24 años de edad y las frecuencias más altas están entre los 23 a 25 años. La frecuencia más alta es de 25 años y la menor es de 20 años; es interesante indicar que la Universidad de Cuenca ha recibido estudiantes de movilidad de 50 años de edad.

Al hablar del tipo de estudiante de movilidad que aplican se categorizó en base a practicantes, que estos pueden ser estudiantes de rotaciones médicas o en Facultades que les permitan realizar sus prácticas pre-profesionales y que puedan ser homologadas en sus instituciones de origen; por otro lado están los estudiantes según el programas específico, que son aquellos que se encuentran dentro de cátedras o en Facultades de la institución para realizar actividades delimitadas y se tienen a los estudiantes que se vinculan a Facultades para realizar un semestre o hasta un año de movilidad estudiantil. El $93 \%$ de los encuestados realizaron su movilidad durante un semestre académico, el $5 \%$ fue la respuesta de estudiantes de prácticas pre profesionales, y el $2 \%$ fueron de estudiantes que aplicaron a programas específicos, como se observa en la Tabla 1.

Tabla 1. Tipo de estudiante

\begin{tabular}{|c|c|}
\hline Tipo de estudiante & Porcentaje \\
\hline Estudiante de programa específico & $2 \%$ \\
\hline Estudiante de semestre completo & $93 \%$ \\
\hline Practicante & $5 \%$ \\
\hline Total & $\mathbf{1 0 0 \%}$ \\
\hline
\end{tabular}

Fuente: Elaboración Propia.

La mayor frecuencia de datos está establecida por la contestación de estudiantes de movilidad durante los semestres de septiembre 2015 - febrero 2016 y marzo - julio 2016, dando como resultado una movilidad mayor en el 2016 que en el 2015, Figura 4. Se consideró pertinente consultar a los estudiantes de movilidad, el nombre de la Universidad emisora y receptora, a más del nombre de las Facultades y carreras de procedencia como de destino, y se ha levantado la información pertinente a la aplicación de su movilidad en base a los criterios de las áreas del conocimiento establecidas por la UNESCO; y se consideró pertinente analizar las Universidades y las áreas del conocimiento dadas por la UNESCO.

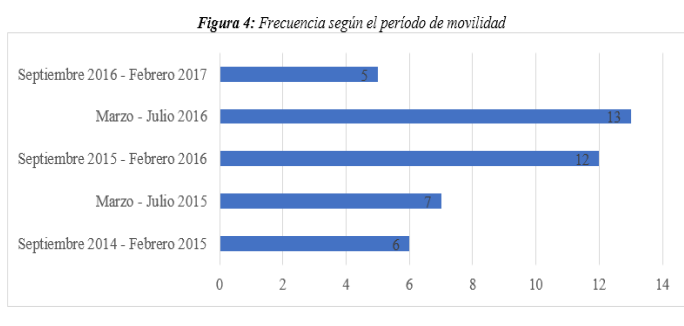

Fuente: Elaboración Propia. 
En relación con las políticas de la DRI, se analizó su estado actual y posterior a ello se colocaron algunas sugerencias, que de ser consideradas podrían ser de mucha utilidad institucional para reforzar en varios ámbitos, siendo el primordial la movilidad; es importante recalcar que al tratarse de temas de internacionalización, siempre se tendrá que apuntar a un beneficio entre las partes y sobre todo en equiparlo con estándares de calidad altos para mejorar particularidades de las instituciones.

La DRI disponía de 7 políticas y acciones que regulaban la función y actividades que regulaban los procesos desarrollados.

1. Fortalecer y promover vínculos de cooperación académica y de trabajo con otras instituciones de educación superior en el extranjero.

2. Promover la participación en redes académicas y de investigación con instituciones de alto prestigio internacional.

3. Incrementar las relaciones de amistad y cooperación con las diversas Embajadas y Consulados.

4. Promover la movilidad estudiantil y docente con diversas universales del extranjero.

5. Cooperar con las Facultades y Departamentos de la Universidad de Cuenca, en temas de internacionalización.

6. Promover e informar sobre oportunidades de becas, cursos o programas que ofertan las instituciones extranjeras.

7. 7. Apoyar en acrecentar la imagen institucional de la Universidad de Cuenca a nivel internacional.

En base a estas políticas, se analizaron y sugirieron cambios que implicaban: dar seguimiento a convenios vigentes y activos con la implícita necesidad de conformar responsables en cada uno de los acuerdos, que figure un marco completo claro en sus actividades para llevar a cabo de manera oportuna la cooperación internacional; que propone una flexible, amigable y coordinada organización; participar activamente en redes y asociaciones para encontrar soluciones a problemáticas sociales en cada una de las localidades, alineado a la consolidación institucional para que la comunidad universitaria pueda brindar un aporte sin olvidar conseguir un plus con la dinámica internacional; trabajar en conjunto con las embajadas, que catapulten actividades y mejoren sus relaciones institucionales, ligando temas culturales, tecnológicos, académicos, migratorios, entre otros; reforzar la coordinación internar y externa, capaz de brindar un soporte previo, durante y después de la movilidad, ya que los indicadores de una participación internacional son beneficiosos para la imagen institucional puertas afueras. Del mismo modo, la comunicación deberá ser fluida ya que la documentación y registro son parte importante al momento de una aplicación; reforzar la dinámica de comunicación, la misma que deberá ser efectiva y con el ánimo de mejorar los procesos que benefician a una correcta internacionalización y va desde los niveles jerárquicos más bajos hasta los más altos; y, finalmente difundir oportunamente opciones de becas, cursos, programas ofertados en el extranjero, basándose en ejes fundamentes como la confidencialidad y veracidad de la información; posicionar de la universidad con el buen uso de la imagen institucional, la misma que está ligada con una identidad más sólida de estudiantes, docentes y cuerpo administrativo de la institución.

\section{RESULTADOS}

Luego de haber revisado las políticas de la DRI, en relación con la movilidad y directamente el conjunto de acciones, es preferible plantear ciertas sugerencias para que puedan ser consideradas para el mejoramiento de la calidad y pertinencia en sus actividades, las mismas que son las siguientes:

Política 1: Identificar, promover y dar seguimiento a la cooperación académica internacional alineada a las necesidades institucionales.

\section{Acciones:}

- Realizar visitas de trabajo a las instituciones internacionales con las que se mantiene una cooperación vigente para afianzar y/o ampliar líneas de trabajo.

- Fomentar acercamientos interinstitucionales con Universidades extranjeras a fin de concretar cooperación en áreas del conocimiento de interés mutuo.

- Plantear la celebración de convenios específicos y marcos con sus respectivos responsables en cada una de las instituciones.

- Promover la cooperación de una movilidad estudiantil, docente y de planta administrativa alineada a las necesidades institucionales.

Política 2: Promover la participación activa de la Universidad de Cuenca en redes académicas y de investigación, reconocidas internacionalmente y que sean de beneficio institucional.

\section{Acciones:}

- Determinar fortalezas de las redes y asociaciones académicas de las cuales la Universidad de Cuenca forma parte.

- Identificar a través de las unidades académicas, redes y/o asociaciones de interés a las que la Universidad de Cuenca pueda afiliarse.

- Establecer cooperación conjunta en proyectos en pos de los intereses generales de las instituciones.

- Incentivar a los miembros de la comunidad universitaria a sacar provecho de las oportunidades brindadas de las redes de las cuales forma parte, si el caso lo amerita.

Política 3: Fortalecer las relaciones de cooperación con Embajadas y Consulados.

Acciones:

- Realizar visitas a Embajadas extranjeras que funcionan en Ecuador, así como a los consulados domiciliados en Cuenca, a fin de buscar su colaboración en actividades propuestas por la Universidad. 
- Establecer líneas de trabajo conjunto con las Embajadas para fortalecer la cooperación internacional.

- Invitar a delegaciones de las Embajadas a dar charlas informativas de convocatorias de programas académicos en sus países.

- Programar actividades culturales a través de los agregados culturales de las embajadas de los países.

- Buscar apoyo para le enseñanza de los idiomas nativos de los países para trabajar en conjunto con la institución.

- Mantener contacto en relación con los procesos migratorios y de visado para estudiantes, docentes y administrativos.

Política 4: Promover la movilidad estudiantil y docente en los diferentes programas y convocatorias a nivel nacional e internacional.

\section{Acciones:}

- Brindar charlas informativas con apoyo de representantes de instituciones u organismos internacionales promoviendo la movilidad en distintas áreas del conocimiento a nivel internacional.

- Promocionar y difundir por medios de comunicación masivos la información de convocatorias y programas de estudio en el extranjero.

- Apoyar en el proceso de movilidad entrante y saliente a estudiantes y docentes desde y hacia la Universidad de Cuenca.

- Apoyar en el proceso de inserción de estudiantes de pregrado y postgrado para programas de movilidad.

- Consensuar con las autoridades el número determinado de plazas con beca completa para recepción de estudiantes extranjeros para movilidad de un semestre, bajo acuerdos de reciprocidad, a fin de poder enviar estudiantes de la Universidad de Cuenca becados al extranjero.

Política 5: Cooperar con las Facultades y Departamentos de la Universidad de Cuenca, en temas de internacionalización.

\section{Acciones:}

- Brindar información oportuna a las Facultades de la Universidad de Cuenca, sobre convenios de cooperación que se mantienen vigentes.

- Realizar charlas o conferencias direccionadas a las Facultades, con las diversas oportunidades en el extranjero.

- Apoyar los procesos de firma de convenios entre las unidades académicas con instituciones extranjeras.

- Coordinar reuniones de trabajo entre instituciones interesadas en trabajar conjuntamente con las unidades académicas de la Universidad de Cuenca.

Política 6: Promover e informar a la comunidad universitaria sobre oportunidades de becas, cursos o programas que ofertan las instituciones extranjeras.
Acciones:

- Mantener comunicación interna vía correo electrónico y medios de comunicación masiva, la oferta de oportunidades para estudios en el extranjero.

- Realizar conferencias con la participación de organizaciones extranjeras, para promocionar las opciones de estudios.

- Apoyar a los docentes, estudiantes y personal administrativo para conseguir cupos en seminarios, cursos, talleres a los que se deseen asistir.

Política 7: Apoyar en acrecentar la imagen institucional de la Universidad de Cuenca a nivel internacional.

Acciones:

- Participar en ferias y congresos en el extranjero, para dar a conocer las fortalezas de la Universidad de Cuenca en sus áreas del conocimiento.

- Mantener comunicación con Universidades extranjeras para dar a conocer sobre oportunidades de estudio en la Universidad de Cuenca.

- Mantener una buena relación con embajadas, instituciones y organizaciones extranjeras.

\section{DISCUSIÓN}

La Universidad de Cuenca, deberá considerar sus políticas y normativas de la Dirección de Relaciones Internacionales, y de ser posible el reglamento general de la institución, para clarificar aspectos tanto para administrativos, docentes y estudiantes tanto nacionales como internacionales, siendo el centro motor de la cooperación internacional, la movilidad estudiantil, esto quiere decir que la DRI debe establecer parámetros para que la movilidad sea una actividad dinámica utilizando todos los recursos y oportunidades para una adecuada ejecución de la movilidad en todas sus etapas; buscando formas de financiamiento para incrementar la movilidad desde la UC hacia otras instituciones extranjeras, a más de ser inclusivo con áreas del concomimiento que no están realizando movilidad internacional.

Los convenios de cooperación, la participación en asociaciones, las membresías en redes internacionales son piezas clave de la internacionalización con componentes de movilidad estudiantil, docente, etc.

\section{CONCLUSiones}

La movilidad estudiantil internacional en la Universidad de Cuenca tuvo un número de 112 postulantes en el año 2015 y 97 postulaciones en el 2016, con lo cual se puede identificar dos escenarios; el primero, existió una activación de mayor número de convenios de movilidad estudiantil, acceso a becas de movilidad basados en términos de reciprocidad a través de asociaciones y redes de las que la Universidad de Cuenca es parte; y segundo, la disminución de postulaciones en el 2016 debido a factores externos ya que el costo de las visas emitidas en el 2015 para las aplicaciones del siguiente año fue muy 
elevado y como consecuencia el bajo ingreso de estudiantes extranjeros de manera regular al Ecuador; a pesar de ello, la movilidad estudiantil está tomando un ritmo de participación estable para incrementar lazos de cooperación y mejoramiento de la calidad de la educación superior.

Se destaca que, las políticas y reglamentación de movilidad estudiantil, sí son un factor de influencia en la toma de decisión de intercambio; las políticas y reglamentos internos son un factor determinante para asegurar la homologación de créditos académicos a su retorno a la institución de origen, enfatizando la importancia de comunicación y socialización de todo lo concerniente a los procesos de movilidad; del mismo modo, las áreas del conocimiento de mayor aplicación fueron: salud y servicios sociales con un 37,80\%; y el área de ciencias sociales, educación comercial y derecho con un 21,53\% de los datos obtenidos se observa que se debe reforzar las otras áreas tales como las de educación, humanidades y artes y servicio. Adicional a ello, se realizaron recomendaciones para el mejoramiento y ampliar la visión institucional, junto con algunos avances en los procesos de movilidad estudiantil, asignación de becas y aceptación institucional, que se encuentran inmersas en las políticas de la dependencia.

\section{AgradeCIMIENTOS}

La investigación fue posible gracias al apoyo de la Dirección de Relaciones Internacionales de la Universidad de Cuenca, a los estudiantes que colaboraron con las encuestas y al proceso de mejora continua a la que se compromete la institución.

\section{REFERENCIAS BIBLIOGRÁFICAS}

IESALC (2017). Enero 2017 / Boletín Proyecciones UNESCO - IESALC.

Muñoz Varela, M. (2017). Política y estrategias de movilidad académica y estudiantil en la Universidad de Costa Rica. San José.

Organización de Naciones Unides (1948). Declaratoria Universal de Derechos Humanos. París.

Palma, J. J. G. (2013). Movilidad estudiantil internacional y cooperación educativa en el nivel superior de educación1. TEMAS/TEMAS.

Secretaria de Educación Superior, Ciencia, T. e. I. (2013). La SENESCYT promueve la movilidad académica y científica en el Ecuador - Senescyt - Secretaría de Educación Superior, Ciencia, Tecnología e Innovación.

Universidad de Cuenca (2016). Informe de Gestión 20112016. Cuenca.

Vizcardo, J. P. et al. (2004). Carta dirigida a los españoles americanos. Fondo De Cultura Economica USA. 